\title{
Different Kinds of Singular and Nonsingular Exact Traveling Wave Solutions of the Kudryashov-Sinelshchikov Equation in the Special Parametric Conditions
}

\author{
Can Chen, Weiguo Rui, and Yao Long \\ College of Mathematics of Honghe University, Mengzi, Yunnan 661100, China \\ Correspondence should be addressed to Yao Long; longyaoteacher@yahoo.com.cn
}

Received 18 March 2013; Accepted 16 May 2013

Academic Editor: Sotiris Ntouyas

Copyright (C) 2013 Can Chen et al. This is an open access article distributed under the Creative Commons Attribution License, which permits unrestricted use, distribution, and reproduction in any medium, provided the original work is properly cited.

In this paper, by using the integral bifurcation method, we studied the Kudryashov-Sinelshchikov equation. In the special parametric conditions, some singular and nonsingular exact traveling wave solutions, such as periodic cusp-wave solutions, periodic loop-wave solutions, smooth loop-soliton solutions, smooth solitary wave solutions, periodic double wave solutions, periodic compacton solutions, and nonsmooth peakon solutions are obtained. Further more, the dynamic behaviors of these exact traveling wave solutions are investigated. It is found that the waveforms of some traveling wave solutions vary with the changes of parameters.

\section{Introduction}

The investigation of the traveling wave solutions for nonlinear evolution equations plays an important role in mathematical physics. For example, the phenomena of wave motion observed in fluid dynamics, plasma, and elastic media are usually described by the solitary wave, kink wave, peakon, compacton, and loop soliton. In fact, lots of physical models have solitary wave solutions, kink wave solutions, peakon solutions, compacton solutions, and loop-soliton solutions. Lots of new traveling wave solutions and phenomena of wave motion are discovered continually by many researchers on investigating exact solutions of different kinds of nonlinear evolution equations. Recently, Kudryashov and Sinelshchikov [1] introduced the following equation:

$$
u_{t}+\alpha u u_{x}+u_{x x x}-\epsilon\left(u u_{x x}\right)_{x}-\beta u_{x} u_{x x}-v u_{x x}-\delta\left(u u_{x}\right)_{x}=0,
$$

where $\alpha, \epsilon, \beta, \nu$, and $\delta$ are real parameters. Equation (1) describes the pressure waves in the liquid with gas bubbles taking into account the heat transfer and viscosity [1]. We call (1) Kudryashov-Sinelshchikov equation. When $\epsilon=\beta=$ $\delta=0$, (1) becomes classical KdV-Burgers (Burgers-KdV) equation; see [2-7] and the references cited therein. When $\epsilon=\beta=\nu=\delta=0$, (1) becomes famous KdV equation. Very recently, when parameters $v=\delta=0$, in [8-11], the authors studied the special case of (1) as follows:

$$
u_{t}+\alpha u u_{x}+u_{x x x}-\left(u u_{x x}\right)_{x}-\beta u_{x} u_{x x}=0 .
$$

In [8], Ryabov found four families of solitary wave solutions of (2) when $\beta=-3$ or $\beta=-4$. In [9], Li and Chen discussed the existence of different kinds of traveling wave solutions by using the approach of dynamical system, according to different phase orbits of the traveling system of (2); twenty-six kinds of exact traveling wave solutions are obtained under the parametric conditions $\beta=-3,-4$ and $\beta=1,2$. In [10], He et al. discussed the bifurcations of phase portraits and investigated exact traveling wave solutions of (2) under $\beta=-3,1,2$. In [11], He et al. investigated periodic loop solutions of (2) and discussed the limit forms of these periodic loop solutions focusing on the case $\beta=2$. In the paper [12], Randrüüt studied (2) under the transformation $b=2+\beta$; in the cases $b<0, b=0$, and $b>0$ (i.e., $\beta<-2, \beta=-2$, and $\beta>-2$ ), Randrüüt obtained some exact traveling wave solutions and discussed their dynamical behaviors; some interesting phenomena of traveling waves are successfully explained. Particularly, when $b>2$ (i.e., $\beta>0$ ), a kind of new periodic wave solution which is called meandering solution was obtained by Randrüut in this paper. 
However, many singular and nonsingular exact traveling wave solutions of Kudryashov-Sinelshchikov equation were not obtained yet in these references [8-12].

In this paper, we will study (2) again by using the integral bifurcation method [13, 14]; the integral bifurcation method possessed some advantages of the bifurcation theory of the planar dynamic system [15-19] and auxiliary equation method; it is easily combined with some transformations $[20,21]$ and useful for many nonlinear partial differential equations (PDEs) including some higher order equations of $\mathrm{KdV}$ type, such as the higher order $\mathrm{KdV}$ equation of neglecting the highest order infinitesimal term [22]. So, by using this method, we will obtain some new traveling wave solutions of (1), which are different from the results in [8-12]. Some interesting phenomena will be presented.

The rest of this paper is organized as follows: in Section 2, we will derive two-dimensional planar system which is equivalent to (2) and give its first integral equation. In Section 3, by using the integral bifurcation method, we will obtain some new traveling wave solutions of (2) and discuss their dynamic properties.

\section{The 2-Dimensional Planar Dynamical System of (2)}

Making a transformation $u(t, x)=\phi(\xi)+\lambda$ with $\xi=k x-$ $c t$, (2) can be reduced to the following ordinary differential equation:

$$
-c \phi^{\prime}+\alpha(\phi+\lambda) \phi^{\prime}+\phi^{\prime \prime \prime}-\left[(\phi+\lambda) \phi^{\prime \prime}\right]^{\prime}-\beta \phi^{\prime} \phi^{\prime \prime}=0 .
$$

Integrating (3) with respect to $\xi$, we have

$$
(1-\lambda-\phi) \phi^{\prime \prime}=g+(c-\alpha \lambda) \phi-\frac{1}{2} \alpha \phi^{2}+\frac{1}{2} \beta\left(\phi^{\prime}\right)^{2},
$$

where $g$ is an integral constant. Let $d \phi / d \xi=y$. Thus, (4) can be rewritten as the following 2-dimensional planar system:

$$
\frac{d \phi}{d \xi}=y, \quad \frac{d y}{d \xi}=\frac{g+(c-\alpha \lambda) \phi-(1 / 2) \alpha \phi^{2}+(1 / 2) \beta y^{2}}{(1-\lambda-\phi)}
$$

which is a singular system. The system (5) has the following first integral:

$$
H(\phi, y) \equiv(-1+\lambda+\phi)^{\beta}\left[y^{2}+\frac{a_{0}+a_{1} \phi+a_{2} \phi^{2}}{\beta(\beta+1)(\beta+2)}\right]=h,
$$

where $h$ is integral constant and $a_{0}=2 g(\beta+1)(\beta+2)-2(\lambda-$ 1) $[c(\beta+2)-\alpha \lambda(\beta+1)-\alpha], a_{1}=2(c-\alpha \lambda) \beta^{2}+2 \beta[2 c-\alpha(\lambda+1)]$, and $a_{2}=-\alpha \beta(\beta+1)$. Particularly, when $\beta_{1}=1$, (6) becomes

$$
\begin{aligned}
H(\phi, y) \equiv & (-1+\lambda+\phi) \\
& \times\left[y^{2}+\frac{1}{3} \alpha \phi^{3}+(c-\alpha) \phi^{2}+2 g \phi\right]=h .
\end{aligned}
$$

And when $\beta=2$, (6) becomes

$$
\begin{aligned}
H(\phi, y) \equiv & (-1+\lambda+\phi)^{2} y^{2}-\frac{1}{4} \alpha \phi^{4}+\frac{2 c-3 \alpha \lambda+\alpha}{3} \phi^{3} \\
& +[g+(c-\alpha \lambda)(-1+\lambda)] \phi^{2}+2 g(-1+\lambda) \phi=h .
\end{aligned}
$$

Obviously, (7) is very simpler than (8), that is, the case of $\beta=$ 1 is very simpler than the case of $\beta=2$. Thus we will only discuss the case of $\beta=2$; the case of $\beta=1$ can be similarly discussed, we omit the discussions of case $\beta=1$ in this paper. It is easy to find that the derivative $d y / d \xi$ in the right side of (5) is not defined when $1-\lambda-\phi=0$. Therefore, we make a transformation as follows:

$$
d \xi=(1-\lambda-\phi) d \tau,
$$

where $\tau$ is a parameter. Under the transformation (9) and $\beta=$ 2, (5) becomes the following regular system:

$$
\begin{gathered}
\frac{d \phi}{d \tau}=(1-\lambda-\phi) y, \\
\frac{d y}{d \tau}=g+(c-\alpha \lambda) \phi-\frac{1}{2} \alpha \phi^{2}+y^{2} .
\end{gathered}
$$

Obviously, (8) can be rewritten as follows:

$$
\begin{aligned}
y^{2}= & \frac{1}{(\phi+\lambda-1)^{2}} \\
& \times\left[\frac{\alpha}{4} \phi^{4}-\frac{2(c-\alpha \lambda)-\alpha(\lambda-1)}{3} \phi^{3}\right. \\
& \left.\quad-(g+(c-\alpha \lambda)(\lambda-1)) \phi^{2}-2 g(\lambda-1) \phi+h\right] .
\end{aligned}
$$

\section{Exact Traveling Wave Solutions of (2)}

In this section, we will investigate exact traveling wave solutions of (2) by using its first integral equation (11).

Case 1. When $\lambda=(2 c+\alpha) / 3 \alpha, g=0$, (11) can be reduced to

$$
y= \pm \frac{\sqrt{P \phi^{4}+Q \phi^{2}+R}}{\phi+\lambda-1}
$$

where $P=(1 / 4) \alpha, Q=-8(c-\alpha)^{2} / 9 \alpha$, and $R=h$. Substituting (12) into the first equation of (10) yields

$$
\frac{d \phi}{d \tau}= \pm \sqrt{P \phi^{4}+Q \phi^{2}+R}
$$

By using (13) and (9), we will obtain different kinds of exact traveling wave solutions of (2); see the following discussion.

(i) If $-10<c<-4-2 \sqrt{2}$ or $-4+3 \sqrt{2}<c<2$ and $\alpha=$ $-4, m=(1 / 3) \sqrt{-(1 / 2) c^{2}-4 c+10}, h=(-1 / 18)\left(c^{2}+\right.$ $8 c-2)$, then $P=-1, Q=2-m^{2}$, and $R=m^{2}-1$. 


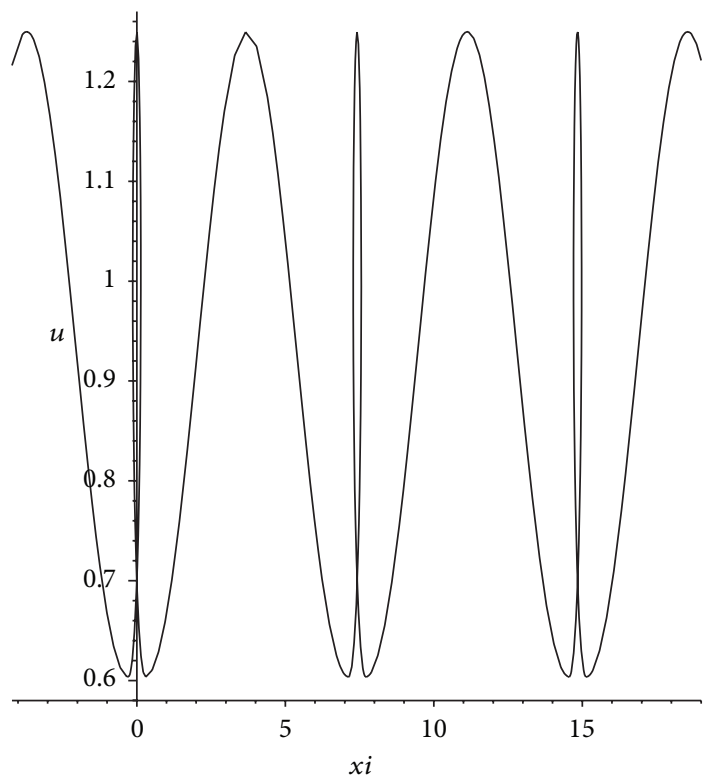

FIGURE 1: The profile of periodic loop-wave solution (14) for $\alpha=-4, c=0.5$.

Under these conditions, by using (13) and (9), we obtain a periodic wave solution of (2) as follows:

$u=\lambda+\operatorname{dn}(\tau, m), \quad \xi=(1-\lambda) \tau-\arcsin (\operatorname{sn}(\tau, m))$.

The profile of solution (14) is a periodic loop wave, which is shown in Figure 1.

(ii) If $-2-\sqrt{34} / 2<c<-2+\sqrt{34} / 2$ and $m=(1 / 34)$ $\sqrt{1462-136 c \pm 102 \sqrt{-2 c^{2}-8 c+9}}, \alpha=(2 / 17)(4 c-$ 9) $\pm(6 / 17) \sqrt{-2 c^{2}-8 c+9}$, then $P=1-m^{2}, Q=$ $2-m^{2}$ and $R=1$. Under these conditions, by using (13) and (9), we obtain a periodic wave solution of (2) as follows:

$$
u=\lambda+\operatorname{sc}(\tau, m),
$$

$\xi=(1-\lambda) \tau-\frac{1}{2 \sqrt{1-m^{2}}} \ln \left[\frac{\operatorname{dn}(\tau, m)+\sqrt{1-m^{2}}}{\operatorname{dn}(\tau, m)-\sqrt{1-m^{2}}}\right]$.

(iii) If $-\infty<c<4-3 \sqrt{2}$ or $4+3 \sqrt{2}<c<\infty$ and $m=$ $(1 / 3) \sqrt{(1 / 2) c^{2}-4 c-1}, \alpha=4, h=(1 / 18)\left(c^{2}-8 c-2\right)$, then $P=1, Q=-\left(1+m^{2}\right)$ and $R=m^{2}$. Under these conditions, by using (13) and (9), we obtain a periodic wave solution of (2) as follows:

$$
\begin{gathered}
u=\lambda+\operatorname{ns}(\tau, m), \\
\xi=(1-\lambda) \tau-\ln \left[\frac{\operatorname{sn}(\tau, m)}{\operatorname{cn}(\tau, m)+\operatorname{dn}(\tau, m)}\right] .
\end{gathered}
$$

(iv) If $4-3 \sqrt{2}<c<4+3 \sqrt{2}$ and $m=(1 / 6) \sqrt{-c^{2}+8 c-2}$, $\alpha=4, h=(1 / 1296)\left(c^{2}-8 c+34\right)\left(c^{2}-8 c-2\right)$, then $P=1, Q=2 m^{2}-1, R=-m^{2}+m^{4}$. Under these conditions, by using (13) and (9), we obtain a periodic wave solution of (2) as follows:

$$
\begin{gathered}
u=\lambda+\mathrm{ds}(\tau, m), \\
\xi=(1-\lambda) \tau-\ln \left[\frac{\operatorname{sn}(\tau, m)}{1+\mathrm{cn}(\tau, m)}\right] .
\end{gathered}
$$

(v) If $1-3 \sqrt{2} / 2<c<-1 / 2$ or $5 / 2<c<1+3 \sqrt{2} / 2$ and $m=(1 / 3) \sqrt{-4 c^{2}+8 c+14}, \alpha=1, h=(1 / 81)\left(2 c^{2}-\right.$ $4 c-7)^{2}$, then $P=1 / 4, Q=(1 / 2)\left(m^{2}-2\right)$, and $R=(1 / 4) m^{4}$. Under these conditions, by using (13) and (9), we obtain two periodic wave solutions of (2) as follows:

$$
\begin{aligned}
u=\lambda & +\operatorname{ns}(\tau, m)+\mathrm{ds}(\tau, m), \\
\xi= & (1-\lambda) \tau-\ln \left[\frac{\operatorname{sn}(\tau, m)}{\operatorname{cn}(\tau, m)+\operatorname{dn}(\tau, m)}\right] \\
& -\ln \left[\frac{\operatorname{sn}(\tau, m)}{1+\operatorname{cn}(\tau, m)}\right], \\
u=\lambda & +\operatorname{ns}(\tau, m)-\operatorname{ds}(\tau, m), \\
\xi= & (1-\lambda) \tau-\ln \left[\frac{\operatorname{sn}(\tau, m)}{\operatorname{cn}(\tau, m)+\operatorname{dn}(\tau, m)}\right] \\
& +\ln \left[\frac{\operatorname{sn}(\tau, m)}{1+\operatorname{cn}(\tau, m)}\right] .
\end{aligned}
$$

The waveforms of solution (19) are transformable; the smooth periodic wave becomes the non-smooth periodic cusp wave as parameter $c$ varies; their profiles are shown in Figures 2(a) and 2(b). In fact, this non-smooth periodic cusp wave is a limit form of smooth periodic wave. 


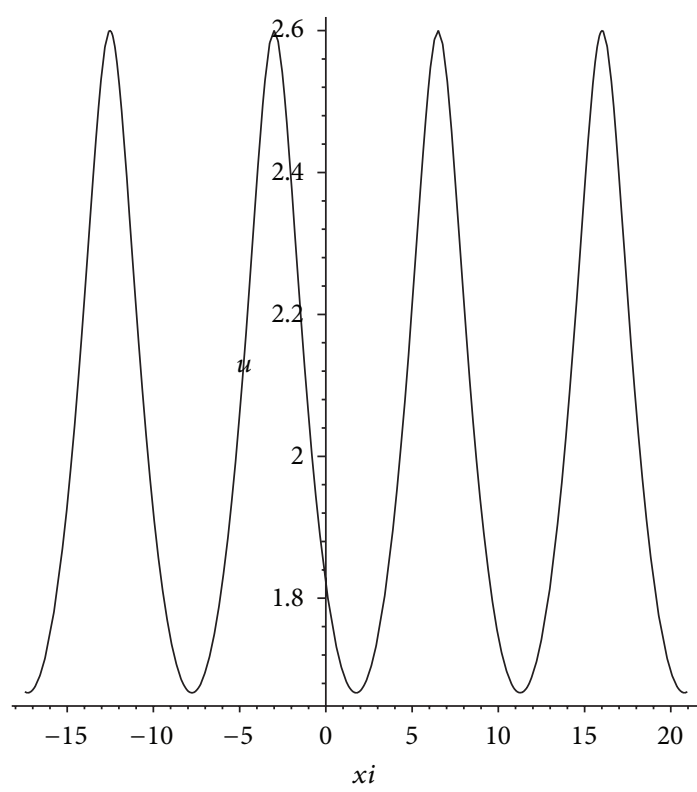

(a) Smooth periodic wave

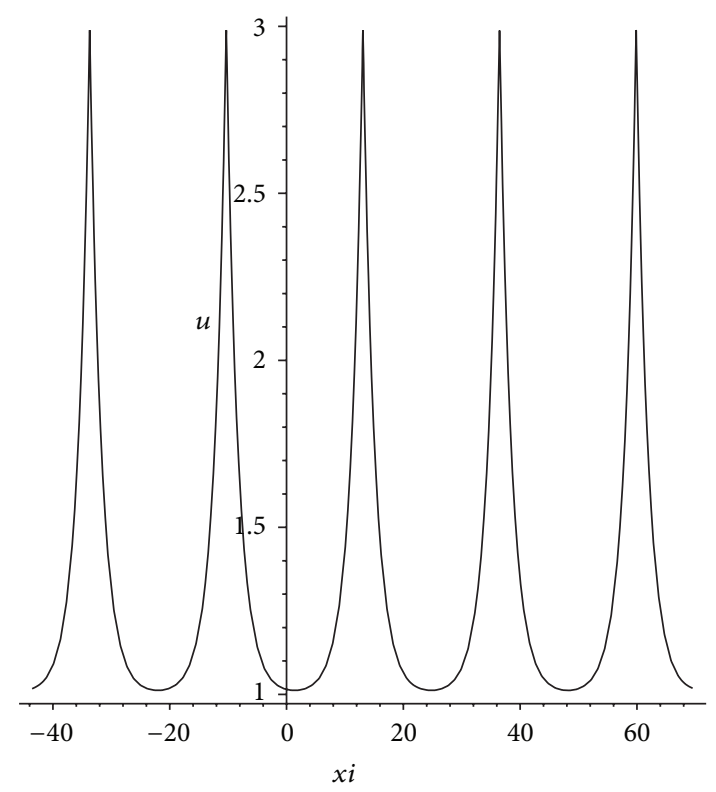

(b) Nonsmooth periodic cusp wave

Figure 2: The profiles of periodic wave solutions (19) for (a) $\alpha=1, c=2.7$, (b) $\alpha=1, c=2.5001$.

Case 2. When $g=0, h=0$, (11) can be reduced to

$$
y=\frac{\sqrt{A \phi^{2}+B \phi^{3}+C \phi^{4}}}{\phi+\lambda-1}
$$

where $A=(c-\alpha \lambda)(\lambda-1), B=\alpha \lambda-(1 / 3)(2 c+\lambda)$, and $C=(1 / 4) \alpha$. Substituting (20) into the first equation of (10) yields

$$
\frac{d \phi}{d \tau}= \pm \sqrt{A \phi^{2}+B \phi^{3}+C \phi^{4}}
$$

By using (21) and (9), we will obtain different kinds of soliton solutions; see the following discussions.

(i) When $A>0, C>0$, by using (21) and (9), we obtain a soliton solution as follows:

$$
\begin{aligned}
& u=\lambda-\frac{A B \operatorname{sech}^{2}((\sqrt{A / 2}) \tau)}{B^{2}-A C[1+\epsilon \tanh ((\sqrt{A / 2}) \tau)]^{2}}, \\
& \xi=(1-\lambda) \tau \\
& \quad+\frac{2}{\epsilon \sqrt{C}} \tanh ^{-1}\left[\frac{\sqrt{A C}}{B}\left(1+\epsilon \tanh \left(\frac{\sqrt{A}}{2} \tau\right)\right)\right],
\end{aligned}
$$

where $\tau$ is a parameter. The solution (22) contains three transformable wave-forms as the parameter $c$ varies; these three wave-forms are dark loop soliton, dark peakon, and smooth-dark soliton, which are shown in Figures 3(a), 3(b), and 3(c). (ii) When $A>0, C<0$, by using (21) and (9), we obtain a soliton solution as follows:

$$
\begin{gathered}
u=\lambda-\frac{A B \operatorname{sech}^{2}((\sqrt{A} / 2) \tau)}{B^{2}-A C[1+\epsilon \tanh ((\sqrt{A} / 2) \tau)]^{2}}, \\
\xi=(1-\lambda) \tau+\frac{2}{\epsilon \sqrt{-C}} \\
\quad \times \arctan \left[\frac{\sqrt{-A C}}{B}\left(1+\epsilon \tanh \left(\frac{\sqrt{A}}{2} \tau\right)\right)\right],
\end{gathered}
$$

where $\tau$ is a parameter. The solution (23) contains two wave-forms, the dark loop soliton, and smooth-bright soliton which are shown in Figure 4.

(iii) When $A<0, C>0$, by using (21) and (9), we obtain a soliton solution as follows:

$$
\begin{gathered}
u=\lambda+\frac{A B \operatorname{csch}^{2}((\sqrt{A} / 2) \tau)}{B^{2}-A C[1+\epsilon \operatorname{coth}((\sqrt{A} / 2) \tau)]^{2}}, \\
\xi=(1-\lambda) \tau+\frac{2}{\epsilon \sqrt{C}} \\
\times \tanh ^{-1}\left[\frac{\sqrt{A C}}{B}\left(1+\epsilon \operatorname{coth}\left(\frac{\sqrt{A}}{2} \tau\right)\right)\right],
\end{gathered}
$$




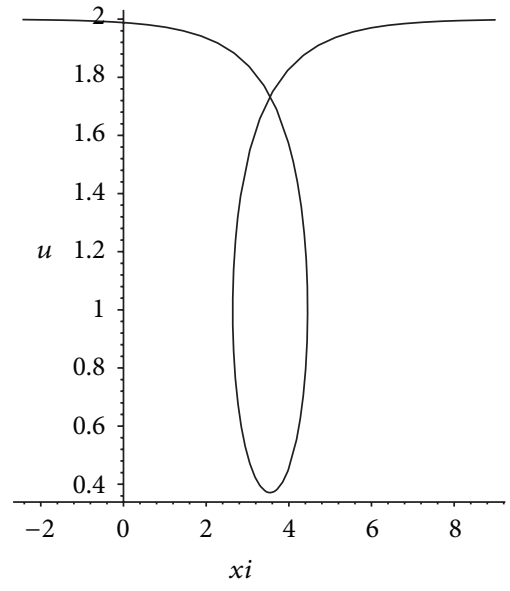

(a) Dark loop soliton

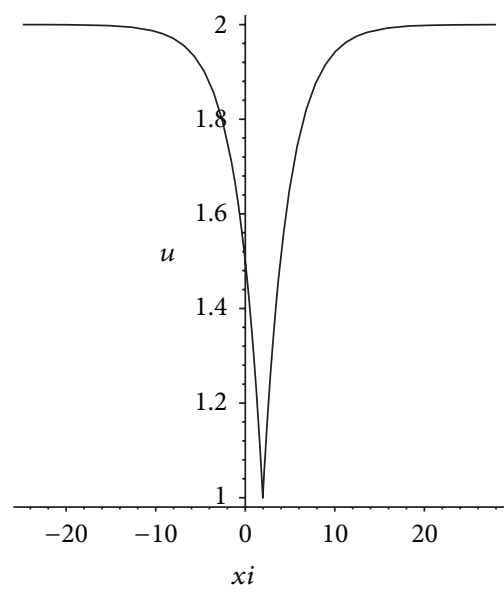

(b) Dark peakon

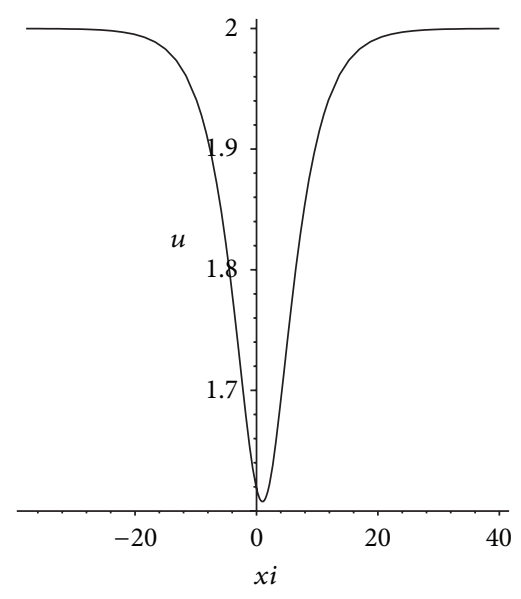

(c) Smooth-dark soliton

FIGURE 3: The profile of soliton solutions (22) for (a) $\alpha=0.5, \lambda=2$, and $c=0.3$, (b) $\alpha=0.5, \lambda=2, c=0.875$, and (c) $\alpha=0.5, \lambda=2$, and $c=0.9375$.

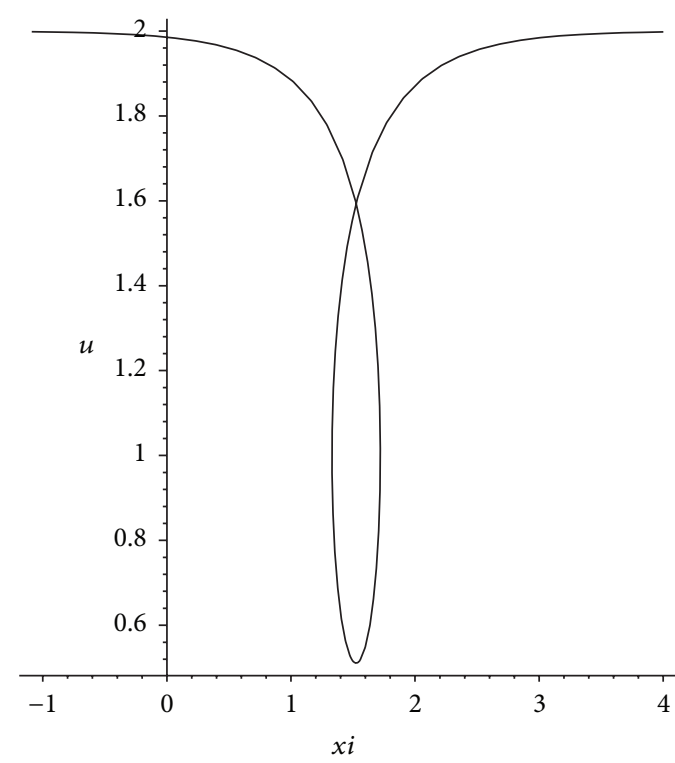

(a) Dark loop soliton

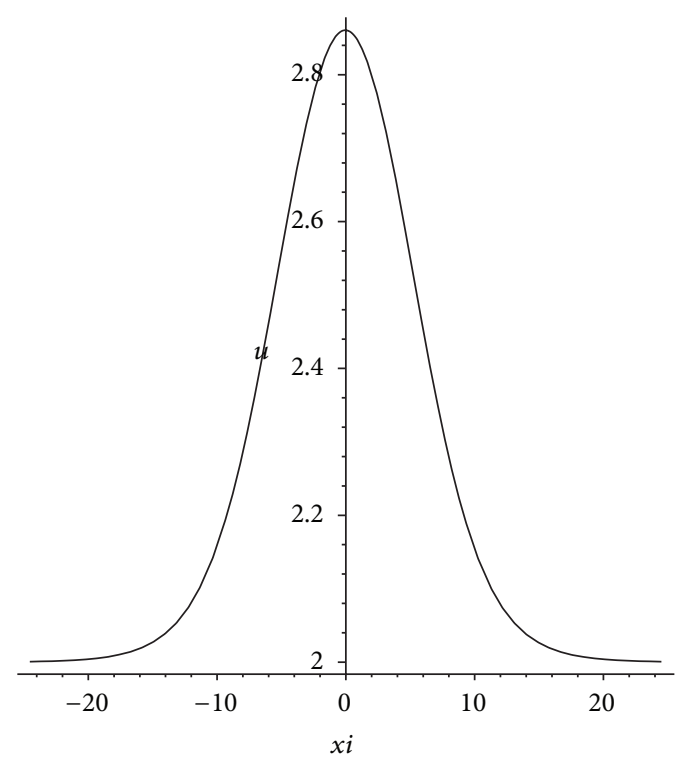

(b) Smooth-bright soliton

FIGURE 4: The profile of soliton solutions (23) for (a) $\alpha=-0.5, \lambda=2$, and $c=-5$ and (b) $\alpha=-0.5, \lambda=2$, and $c=-1.15$.

(iv) When $A<0, C<0$, by using (21) and (9), we obtain a soliton solution as follows:

$$
\begin{gathered}
u=\lambda+\frac{A B \operatorname{csch}^{2}((\sqrt{A} / 2) \tau)}{B^{2}-A C[1+\epsilon \operatorname{coth}((\sqrt{A} / 2) \tau)]^{2}}, \\
\xi=(1-\lambda) \tau+\frac{2}{\epsilon \sqrt{-C}} \\
\times \arctan \left[\frac{\sqrt{-A C}}{B}\left(1+\epsilon \operatorname{coth}\left(\frac{\sqrt{A}}{2} \tau\right)\right)\right] .
\end{gathered}
$$

The solution (25) contains four transformable waveforms as the parameter $c$ varies; these four waveforms are broken wave, broken soliton, dark loop soliton, and smooth-bright soliton, which are shown in Figures 5(a), 5(b), 5(c), and 5(d).

(v) When $A>0, \Delta>0$, and $C>0$, by using (21) and (9), we obtain a soliton solution as follows:

$$
\begin{aligned}
& u=\lambda+\frac{2 A \operatorname{sech}(\sqrt{A} \tau)}{\epsilon \sqrt{\Delta}-B \operatorname{sech}(\sqrt{A} \tau)}, \\
& \xi=(1-\lambda) \tau+\frac{2}{\sqrt{C}} \tanh ^{-1}\left[\frac{B+\epsilon \sqrt{\Delta}}{2 \sqrt{A C}} \tanh \left(\frac{\sqrt{A}}{2} \tau\right)\right] .
\end{aligned}
$$




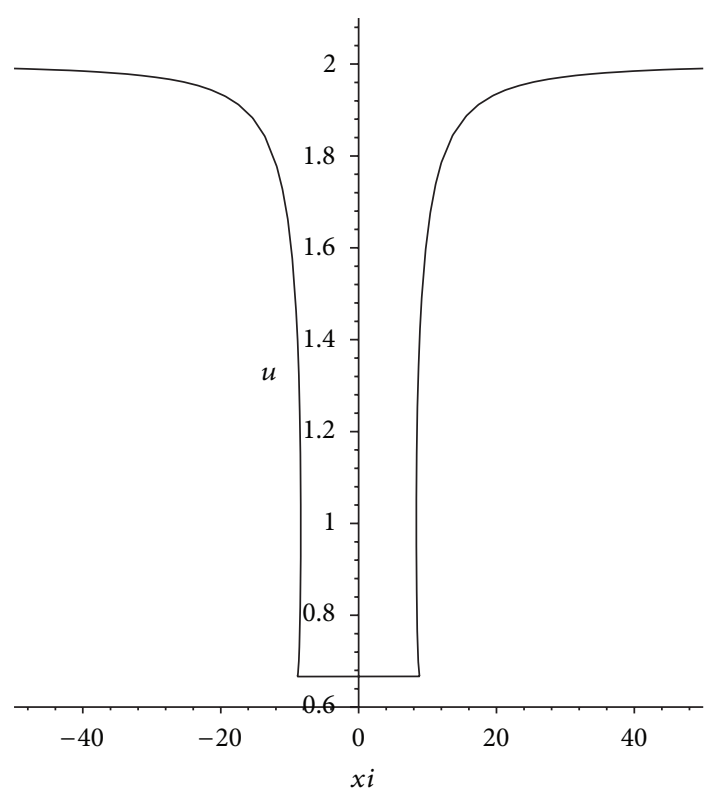

(a) Broken wave

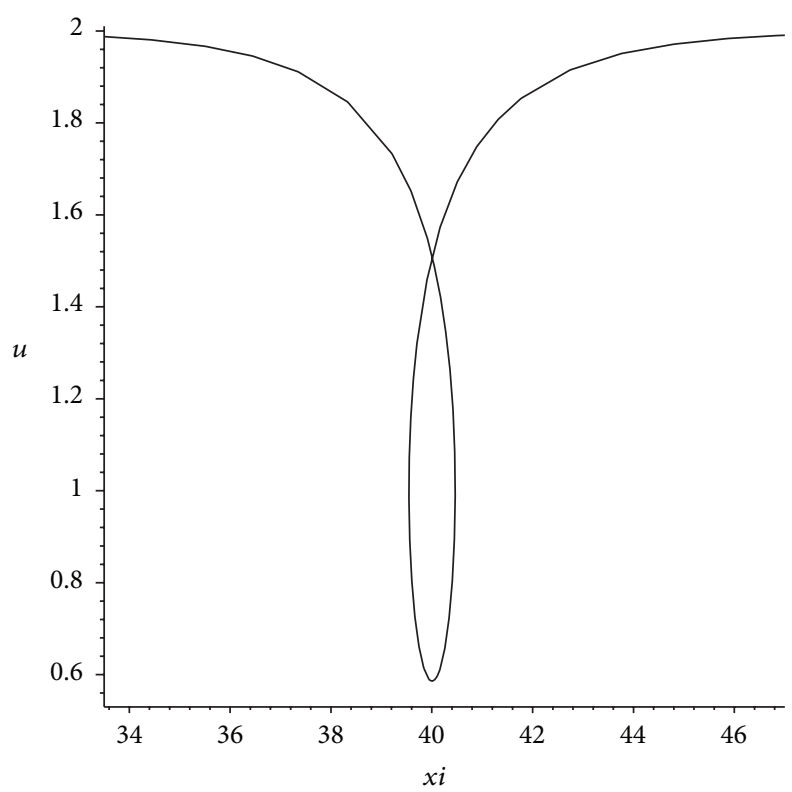

(c) Dark loop soliton

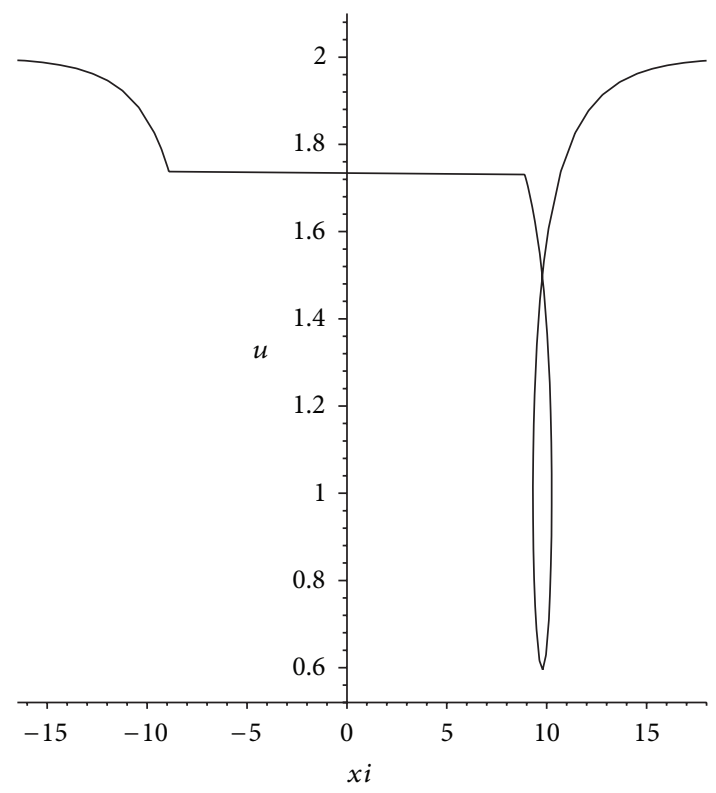

(b) Broken loop soliton

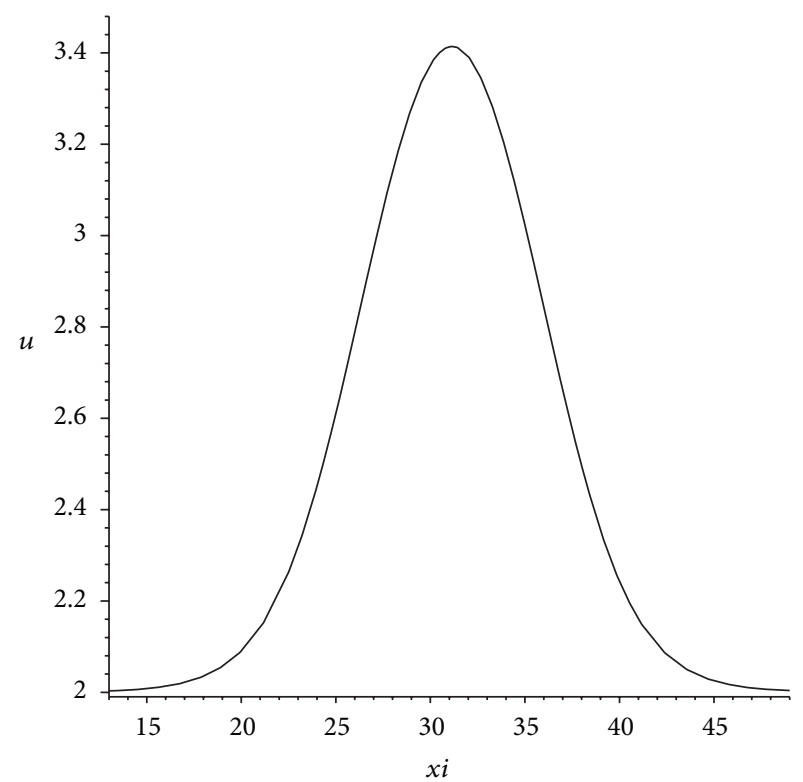

(d) Smooth-bright soliton

FIGURE 5: The profile of soliton solutions (25) for (a) $\alpha=-0.5, \lambda=2$, and $c=-1.0001$, (b) $\alpha=-0.5, \lambda=2$, and $c=-1.2$, and (c) $\alpha=-0.5, \lambda=2$, and $c=-1.25$, and (d) $\alpha=-0.5, \lambda=2$, and $c=-1.25000001$.

(vi) When $A>0, \Delta>0$, and $C<0$, by using (21) and (9), we obtain a soliton solution as follows: (vii) When $A<0, \Delta>0$, and $C<0$, by using (21) and (9), we obtain a soliton solution as follows:

$$
\begin{aligned}
& u=\lambda+\frac{2 A \operatorname{sech}(\sqrt{A} \tau)}{\epsilon \sqrt{\Delta}-B \operatorname{sech}(\sqrt{A} \tau)} \\
& \xi=(1-\lambda) \tau-\frac{2}{\sqrt{-C}} \arctan \left[\frac{B+\epsilon \sqrt{\Delta}}{2 \sqrt{-A C}} \tanh \left(\frac{\sqrt{A}}{2} \tau\right)\right] .
\end{aligned}
$$

$$
\begin{aligned}
& u=\lambda+\frac{2 A \sec (\sqrt{-A} \tau)}{\epsilon \sqrt{\Delta}-B \sec (\sqrt{-A} \tau)} \\
& \xi=(1-\lambda) \tau-\frac{2}{\sqrt{-C}} \arctan \left[\frac{B+\epsilon \sqrt{\Delta}}{2 \sqrt{A C}} \tan \left(\frac{\sqrt{-A}}{2} \tau\right)\right]
\end{aligned}
$$




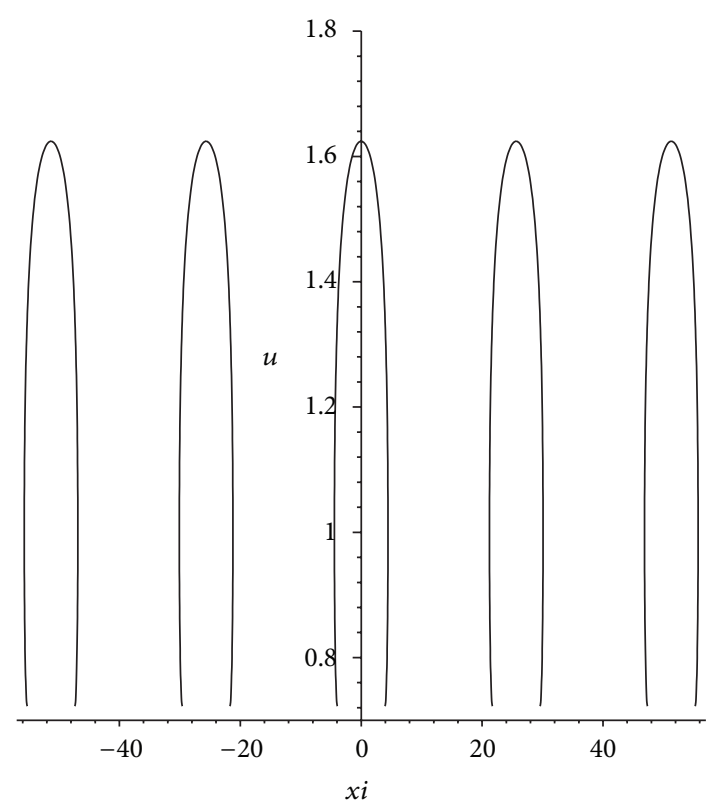

(a) Periodic compacton

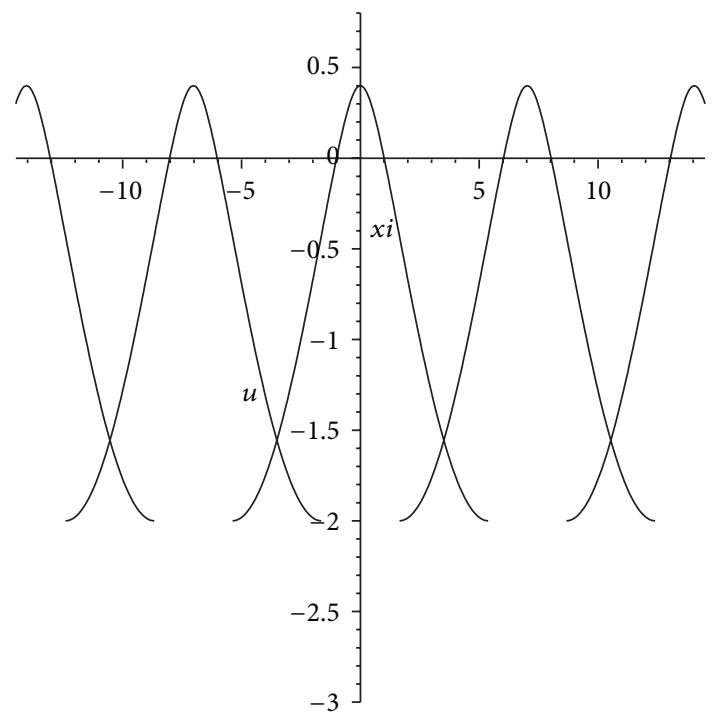

(c) Periodic overlap wave

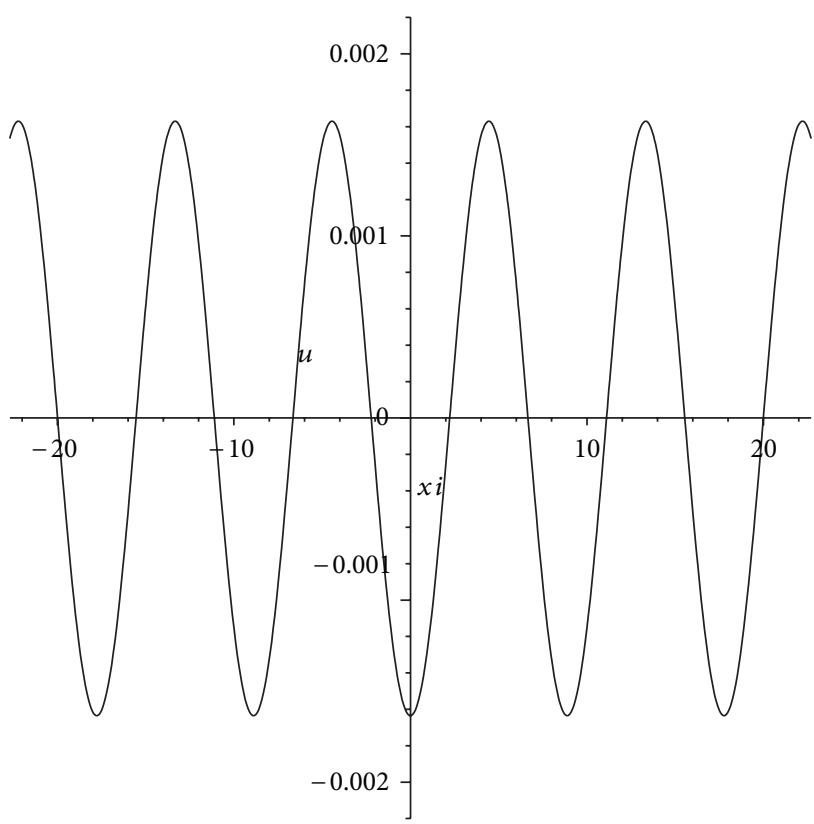

(b) Smooth periodic wave

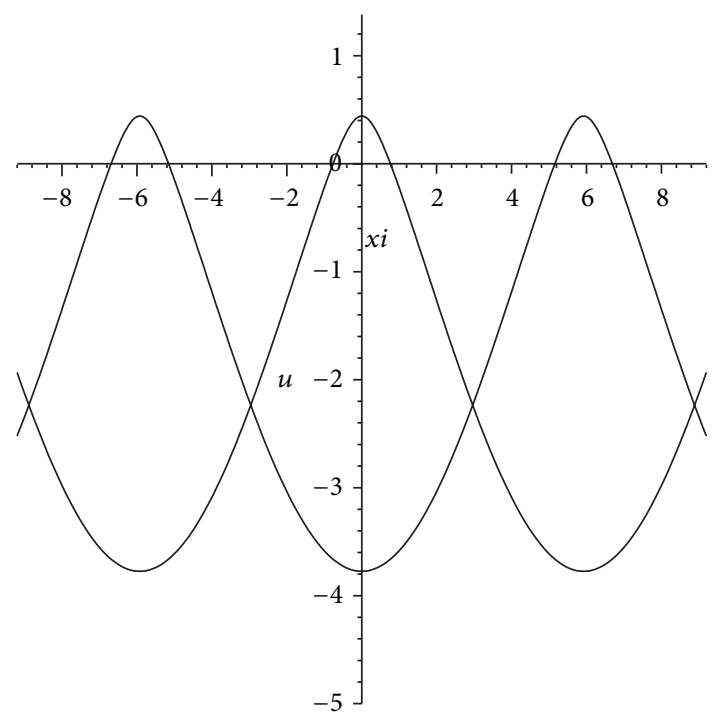

(d) Double periodic overlap wave

FIGURE 6: The profile of soliton solutions (28) for (a) $\alpha=-0.5, \lambda=2$, and $c=-0.94$, (b) $\alpha=-0.5, \lambda=2$, and $c=-0.499999$, (c) $\alpha=-0.5, \lambda=2$, and $c=-0.2$, and (d) $\alpha=-0.5, \lambda=2$, and $c=0.875$.

The solution (28) contains four transformable waveforms as the parameter $c$ varies; these four waveforms are periodic compacton, smooth periodic wave, periodic overlap wave, and double periodic overlap wave, which are shown in Figures 6(a), 6(b), 6(c), and $6(d)$.

(viii) When $A>0, \Delta<0$, and $C>0$, by using (21) and (9), we obtain a soliton solution as follows:

$$
u=\lambda+\frac{2 A \operatorname{csch}(\sqrt{A} \tau)}{\epsilon \sqrt{-\Delta}-B \operatorname{csch}(\sqrt{A} \tau)}
$$

$$
\begin{aligned}
\xi=(1 & -\lambda) \tau+\frac{2}{\sqrt{C}} \\
& \times \tanh ^{-1}\left[\frac{B \tanh ((\sqrt{A} / 2) \tau)+\epsilon \sqrt{-\Delta}}{2 \sqrt{A C}}\right] .
\end{aligned}
$$

(ix) When $A<0, \Delta>0$, and $C<0$, by using (21) and (9), we obtain a soliton solution as follows:

$u=\lambda+\frac{2 A \csc (\sqrt{-A} \tau)}{\epsilon \sqrt{\Delta}-B \csc (\sqrt{-A} \tau)}$ 


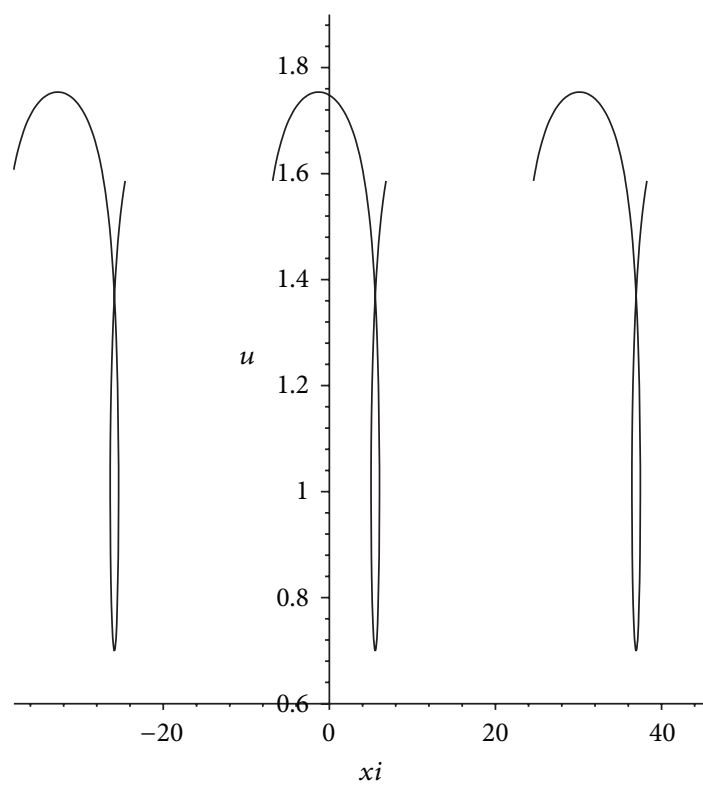

(a) Periodic loop wave

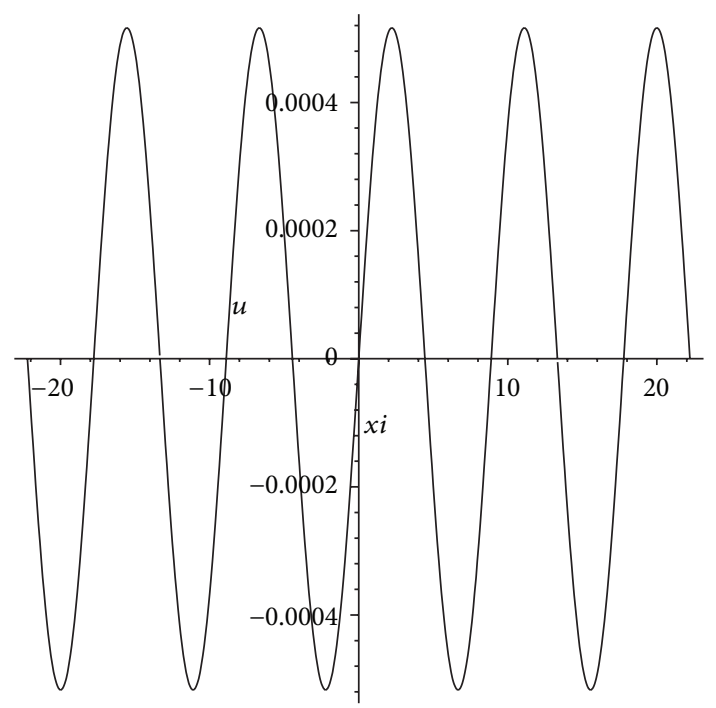

(b) Smooth periodic wave

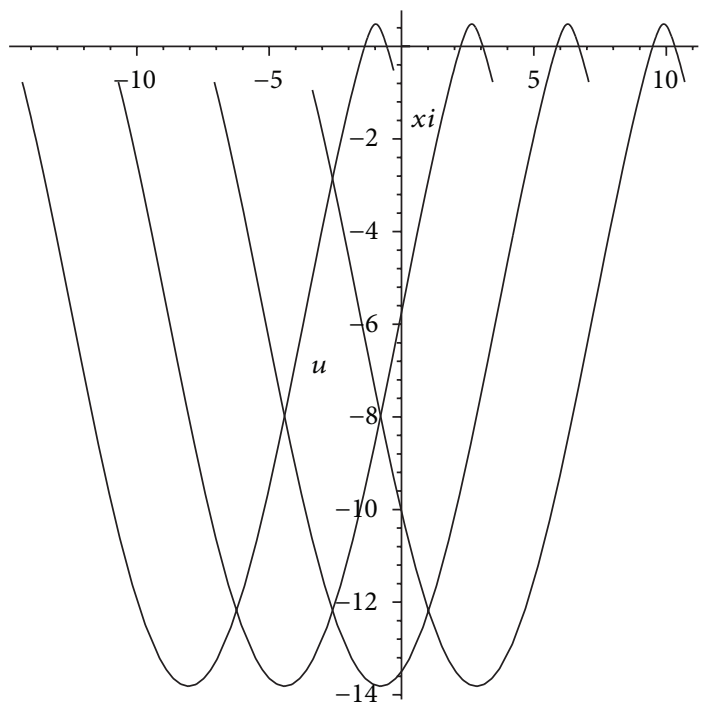

(c) Multiperiodic overlap wave

Figure 7: The profile of soliton solutions (30) for (a) $\alpha=-0.5, \lambda=2$, and $c=-0.96$, (b) $\alpha=-0.5, \lambda=2$, and $c=0.4999999$, and (c) $\alpha=-0.5, \lambda=2$, and $c=2$.

$$
\begin{aligned}
\xi=(1 & -\lambda) \tau+\frac{2}{\sqrt{-C}} \\
& \times \arctan \left[\frac{-B \tan ((\sqrt{-A} / 2) \tau)+\epsilon \sqrt{\Delta}}{2 \sqrt{A C}}\right] .
\end{aligned}
$$

The solution (30) contains three transformable waveforms as the parameter $c$ varies; these three waveforms are periodic loop wave, smooth periodic wave, and multiperiodic overlap wave, which are shown in Figures 7(a), 7(b), and 7(c).

(x) When $A<0, C>0$, by using (21) and (9), we obtain a soliton solution as follows:

$$
u=\lambda-\frac{A \csc ^{2}((\sqrt{-A} / 2) \tau)}{B+2 \epsilon \sqrt{-A C} \cot ((\sqrt{-A} / 2) \tau)},
$$

$\xi=(1-\lambda) \tau-\frac{1}{\epsilon \sqrt{C}} \ln \left|B+2 \epsilon \sqrt{-A C} \cot \left(\frac{\sqrt{-A}}{2} \tau\right)\right|$. 


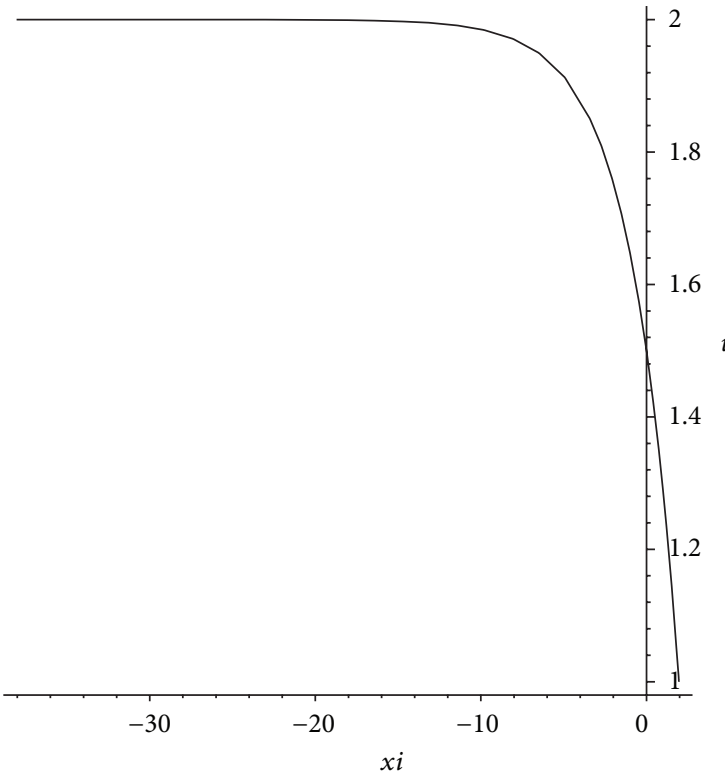

(a) Generalized kink wave

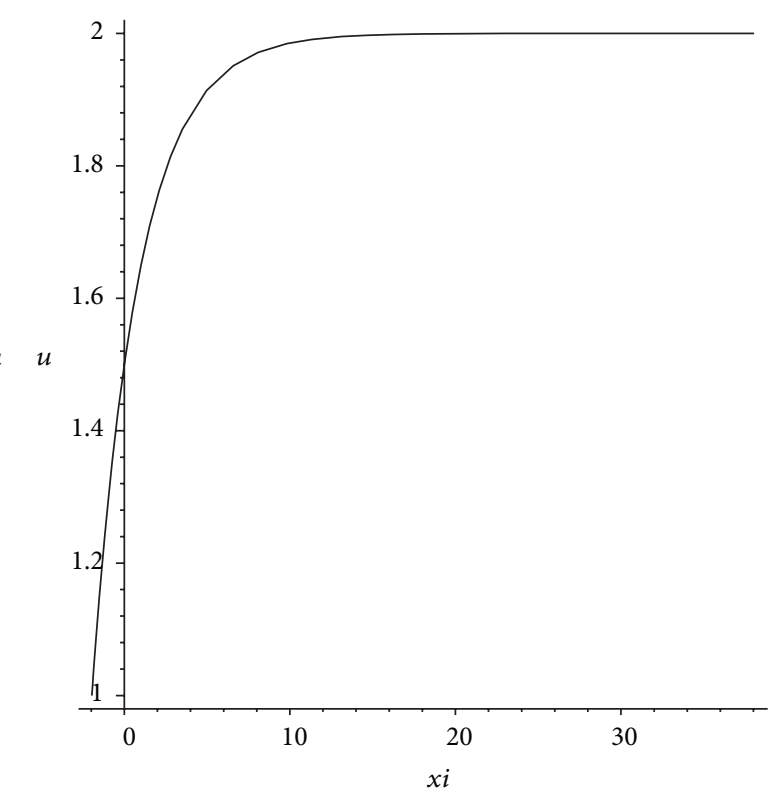

(b) Generalized kink wave

Figure 8: The profiles of soliton solutions (32) for (a) $\alpha=0.5, \lambda=2$, and $c=0.875$, and $\epsilon=-1$ and (b) $\alpha=0.5, \lambda=2, c=0.875$, and $\epsilon=1$.

(xi) When $A>0, \Delta=0$, by using (21) and (9), we obtain a soliton solution as follows:

$$
\begin{gathered}
u=\lambda-\frac{A}{B}\left[1+\epsilon \tanh \left(\frac{\sqrt{A}}{2} \tau\right)\right], \\
\xi=(1-\lambda) \tau+\frac{A}{B} \tau+\frac{2 \epsilon \sqrt{A}}{B} \ln \left|\cosh \left(\frac{\sqrt{A}}{2} \tau\right)\right| .
\end{gathered}
$$

The solution (32) contains two generalized kink waves when $\epsilon= \pm 1$, which are shown in Figures 8(a) and 8(b).

(xii) When $A>0, B=0$, and $C<0$, by using (21) and (9), we obtain a soliton solution as follows:

$$
\begin{gathered}
u=\lambda+\sqrt{-\frac{A}{C}} \operatorname{sech}(\epsilon \sqrt{A} \tau), \\
\xi=(1-\lambda) \tau-\frac{1}{\epsilon \sqrt{-C}} \arctan [\sinh (\epsilon \sqrt{\mathrm{A}} \tau)] . \\
\text { If } A=0, \quad C<0 \\
u=\lambda+\frac{4 B}{(B \tau)^{2}-4 C}, \\
\xi=(1-\lambda) \tau-\frac{2}{\sqrt{-C}} \arctan \left(\frac{B \tau}{2 \sqrt{-C}}\right) .
\end{gathered}
$$

\section{Conclusion}

In this work, by using the integral bifurcation method, we studied Kudryashov-Sinelshchikov equation in the special case $\beta=2$. Some singular and non-singular traveling wave solutions, such as periodic compacton solutions, noncontinuous periodic loop-wave solutions, peakon solutions broken wave solutions, periodic cusp-wave solutions, smooth soliton solutions, continuous periodic loop-wave solutions, and smooth periodic wave solutions are obtained. Some solutions such as (19), (22), (23), (25), (28), and (30) contain multiwave forms; their wave-forms vary accordingly as the parameter $c$ varies. For example, the wave-forms of solution (19) show that the smooth periodic wave becomes the nonsmooth periodic cusp wave as parameter $c$ varies. The waveforms of solution (22) show that the solitary wave becomes solitary cusp wave (peakon) when the value of $c$ arrives at certain limit value; then solitary cusp wave becomes the loop wave when the value of $c$ exceeds this limit value. Indeed, the non-smooth periodic cusp wave can be considered as a kind of long-wave limit of smooth periodic solutions; the loop wave also can be considered as limit wave of smooth solitary wave; these phenomena are very similar to the one in [23]. In [23], according to Braun and Randrüüt's explaination, solitary wave can be considered as the long-wave limit of periodic solutions in $\mathrm{KdV}$ case. By the way, these results which obtained in this paper are different from those in [812].

\section{Acknowledgments}

The authors thank the reviewers very much for their useful comments and helpful suggestions. This research is supported by the National Natural Science Foundation of China (no. 11161020) and the Natural Science Foundations of Yunnan Province (nos. 2011FZ193 and 2013FZ117).

\section{References}

[1] N. A. Kudryashov and D. I. Sinelshchikov, "Nonlinear waves in bubbly liquids with consideration for viscosity and heat 
transfer," Physics Letters A, vol. 374, no. 19-20, pp. 2011-2016, 2010.

[2] M. Wang, "Exact solutions for a compound KdV-Burgers equation," Physics Letters A, vol. 213, no. 5-6, pp. 279-287, 1996.

[3] A. M. Wazwaz, "Travelling wave solutions of generalized forms of Burgers, Burgers-KdV and Burgers-Huxley equations," Applied Mathematics and Computation, vol. 169, no. 1, pp. 639656, 2005.

[4] Z. Feng, "On travelling wave solutions of the Burgers-Kortewegde Vries equation," Nonlinearity, vol. 20, no. 2, pp. 343-356, 2007.

[5] D. Lu, B. Hong, and L. Tian, "New solitary wave and periodic wave solutions for general types of $\mathrm{KdV}$ and $\mathrm{KdV}$-Burgers equations," Communications in Nonlinear Science and Numerical Simulation, vol. 14, no. 1, pp. 77-84, 2009.

[6] R. R. Kumar, K. T. Chong, and J. H. Park, "Exact null controllability of KdV-Burgers equation with memory effect systems," Abstract and Applied Analysis, vol. 2012, Article ID 531659, 20 pages, 2012.

[7] H. Ge and S. Lo, "The KdV-Burgers equation in speed gradient viscous continuum model," Physica A, vol. 391, no. 4, pp. 16521656, 2012.

[8] P. N. Ryabov, "Exact solutions of the Kudryashov-Sinelshchikov equation," Applied Mathematics and Computation, vol. 217, no. 7, pp. 3585-3590, 2010.

[9] J. Li and G. Chen, "Exact traveling wave solutions and their bifurcations for the Kudryashov-Sinelshchikov equation," International Journal of Bifurcation and Chaos, vol. 22, no. 5, Article ID 1250118, 19 pages, 2012.

[10] B. He, Q. Meng, and Y. Long, "The bifurcation and exact peakons, solitary and periodic wave solutions for the Kudryashov-Sinelshchikov equation," Communications in Nonlinear Science and Numerical Simulation, vol. 17, no. 11, pp. 4137-4148, 2012.

[11] B. He, Q. Meng, J. Zhang, and Y. Long, "Periodic loop solutions and their limit forms for the Kudryashov-Sinelshchikov equation," Mathematical Problems in Engineering, vol. 2012, Article ID 320163, 10 pages, 2012.

[12] M. Randrüüt, "On the Kudryashov-Sinelshchikov equation for waves in bubbly liquids," Physics Letters A, vol. 375, pp. 36873692, 2011.

[13] W. Rui, B. He, Y. Long, and C. Chen, "The integral bifurcation method and its application for solving a family of thirdorder dispersive PDEs," Nonlinear Analysis: Theory, Methods \& Applications, vol. 69, no. 4, pp. 1256-1267, 2008.

[14] R. Weiguo, L. Yao, H. Bin, and L. Zhenyang, "Integral bifurcation method combined with computer for solving a higher order wave equation of KdV type," International Journal of Computer Mathematics, vol. 87, no. 1-3, pp. 119-128, 2010.

[15] J. Li and Z. Liu, "Smooth and non-smooth traveling waves in a nonlinearly dispersive equation," Applied Mathematical Modelling, vol. 25, no. 1, pp. 41-56, 2000.

[16] Z. Liu and C. Chen, "Compactons in a general compressible hyperelastic rod," Chaos, Solitons and Fractals, vol. 22, no. 3, pp. 627-640, 2004.

[17] B. Guo and Z. Liu, "Periodic cusp wave solutions and singlesolitons for the b-equation," Chaos, Solitons \& Fractals, vol. 23, no. 4, pp. 1451-1463, 2005.

[18] J. Li, "Exact explicit travelling wave solutions for $(n+1)$ dimensional Klein-Gordon-Zakharov equations," Chaos, Solitons \& Fractals, vol. 34, no. 3, pp. 867-871, 2007.
[19] J. Li and Y. Zhang, "Exact M/W-shape solitary wave solutions determined by a singular traveling wave equation," Nonlinear Analysis: Real World Applications, vol. 10, no. 3, pp. 1797-1802, 2009.

[20] W. Rui and Y. Long, "Integral bifurcation method together with a translation-dilation transformation for solving an integrable 2-component Camassa-Holm shallow water system," Journal of Applied Mathematics, vol. 2012, Article ID 736765, 21 pages, 2012.

[21] W. Rui, "Different kinds of exact solutions with two-loop character of the two-component short pulse equations of the first kind," Communications in Nonlinear Science and Numerical Simulation, vol. 18, no. 10, pp. 2667-2678, 2013.

[22] X. Wu, W. Rui, and X. Hong, "A generalized KdV equation of neglecting the highest order infinitesimal term and its exact traveling wave solutions," Abstract and Applied Analysis, vol. 2013, Article ID 656297, 19 pages, 2013.

[23] M. Braun and M. Randrüüt, "On periodic waves governed by the extended Korteweg-de Vries equation," Proceedings of the Estonian Academy of Sciences, vol. 59, no. 2, pp. 133-138, 2010. 


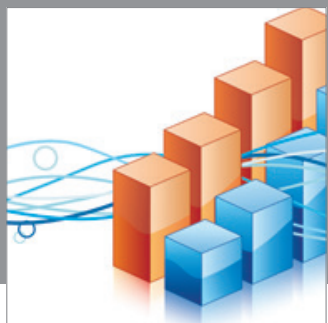

Advances in

Operations Research

mansans

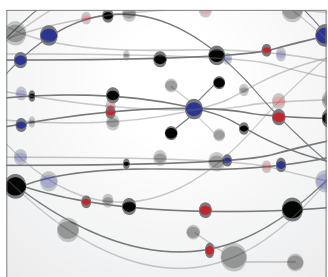

The Scientific World Journal
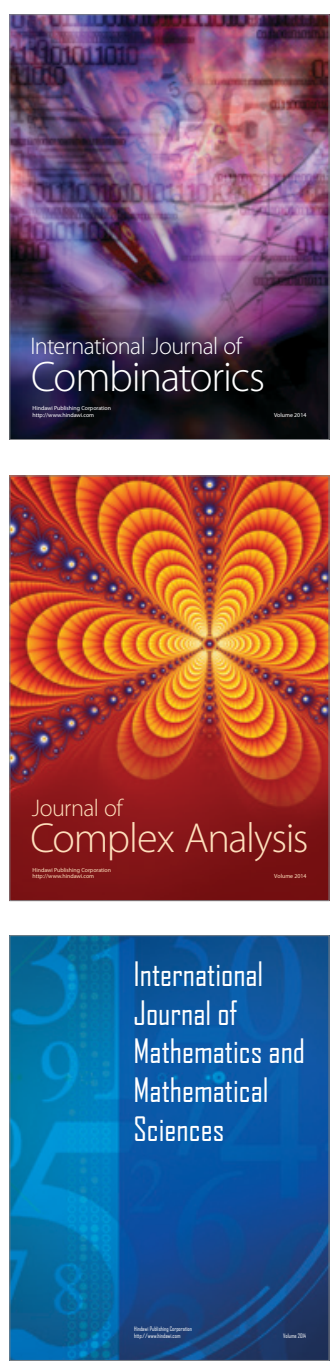
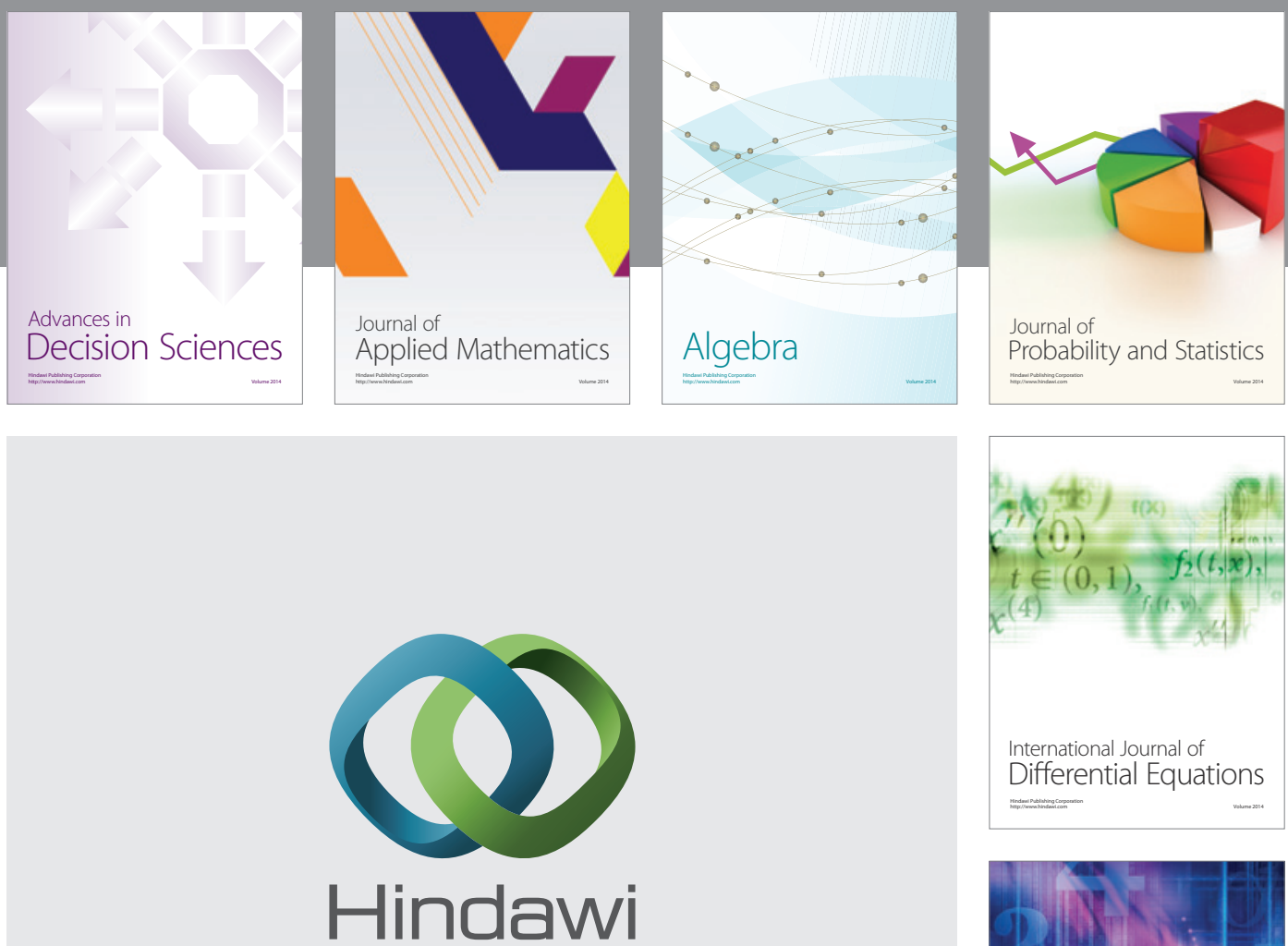

Submit your manuscripts at http://www.hindawi.com
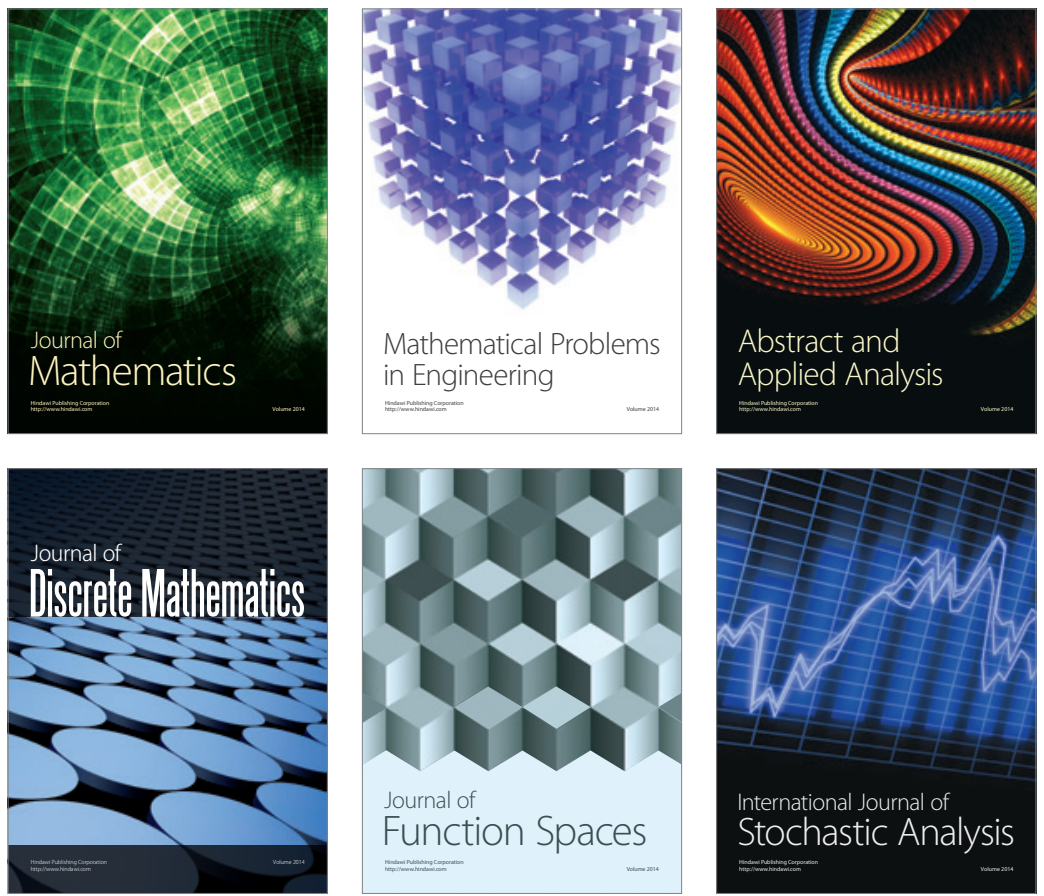

Journal of

Function Spaces

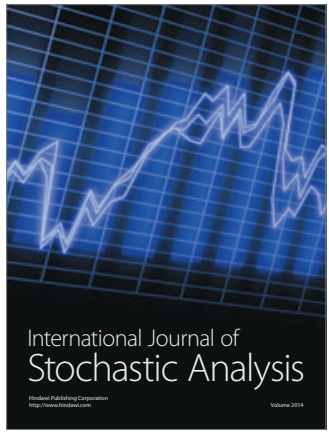

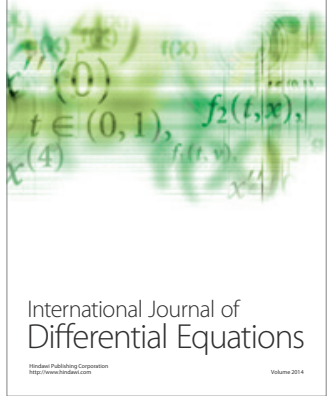
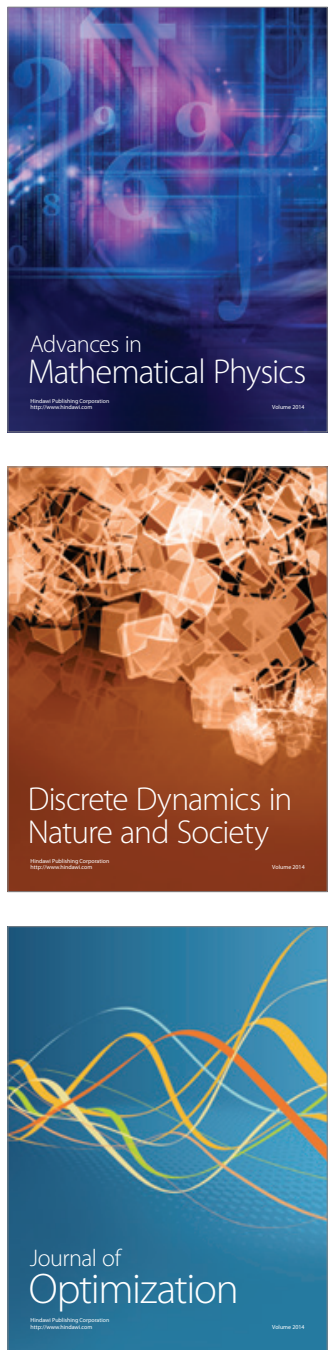